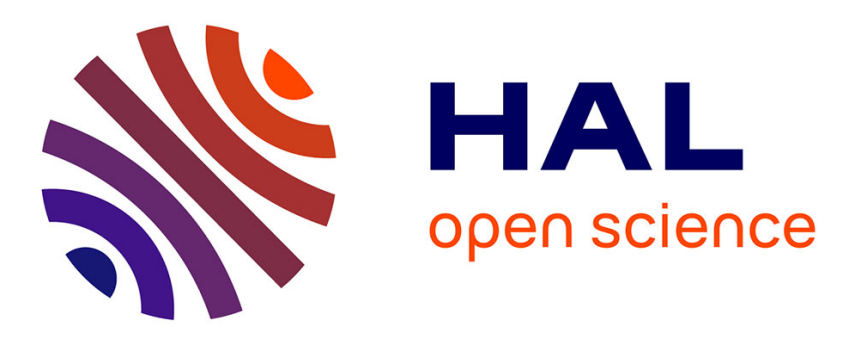

\title{
Fast and Real-Time Sensor-Fault Detection using Shannon's Entropy
}

Nawal Alsaleh, Bilal Daass, Denis Pomorski, Kamel Haddadi

\section{To cite this version:}

Nawal Alsaleh, Bilal Daass, Denis Pomorski, Kamel Haddadi. Fast and Real-Time Sensor-Fault Detection using Shannon's Entropy. 5th IEEE International Conference on Control and Fault-Tolerant Systems (Systol'2021), Oct 2021, Saint-Raphaël, France. 10.1109/SysTol52990.2021.9595413 . hal03508613

\section{HAL Id: hal-03508613 \\ https://hal.archives-ouvertes.fr/hal-03508613}

Submitted on 3 Jan 2022

HAL is a multi-disciplinary open access archive for the deposit and dissemination of scientific research documents, whether they are published or not. The documents may come from teaching and research institutions in France or abroad, or from public or private research centers.
L'archive ouverte pluridisciplinaire HAL, est destinée au dépôt et à la diffusion de documents scientifiques de niveau recherche, publiés ou non, émanant des établissements d'enseignement et de recherche français ou étrangers, des laboratoires publics ou privés. 


\title{
Fast and Real-Time Sensor-Fault Detection using Shannon's Entropy
}

\author{
Nawal Alsaleh, Bilal Daass, Denis Pomorski and Kamel Haddadi
}

\begin{abstract}
In this paper, we aim at improving the change detection techniques by introducing an adaptive thresholding with a sliding time window. In particular, a real-time optimal sliding time window length is implemented without any preliminary learning step as required in conventional sensorfault detection methods. Based on Shannon's entropy, our method improves the change detection techniques using an adaptive thresholding. The technique can be applied by any change detection technique based on the generalized likelihood ratio (GLR). To validate the robustness of our approach, two commonly used change detection techniques are considered: the cumulative sum (Cusum) and the exponentially weighted Moving average (EWMA) control charts. Experimental validation is experimentally shown considering real data in the context of collaborative mobile robots. In addition, this experiment leads to a fault-tolerant fusion methodology based on the use of an extended Kalman filter (EKF).
\end{abstract}

\section{INTRODUCTION}

Change detection is concerned with the identification of data points that differ from those previously observed in the shortest time delay between data change. This technique is also known under the terminology "quickest change detection" [1]. Commonly, change detection techniques are developed through on control charts, implementing statistics based on online tests. These control charts are divided into two categories: memory-less and memory-type [2].

The Shewhart type charts [3] are considered in the category of memory-less control charts. They are entirely based on current information. One of the main drawbacks of the Shewhart control charts is the lack of sensitivity to small changes in the observed signal behavior compared to memorytype charts.

Memory-type control charts include the cumulative sum (Cusum) and the exponentially weighted moving average (EWMA). These methods allow the accumulation of information over time. Indeed, both control charts take into account past and current information to maintain their statistics. This feature of memory-type helps them react quickly to small changes in signal parameters.

The Cusum control chart was initiated in 1954 by Page [4], and has been widely studied in the literature. Examples include Basseville and Nikiforov [5]; Grigg, Farewell and

Nawal Alsaleh is with the University of Lille, CNRS, Centrale Lille, UMR 9189 CRIStAL - Centre de Recherche en Informatique, Signal et Automatique de Lille, F-59000 Lille, France (e-mail: nawal.alsaleh@univlille.fr).

Bilal Daass is with the University of Lille, CNRS, Centrale Lille, UMR 9189 CRIStAL - Centre de Recherche en Informatique, Signal et Automatique de Lille, F-59000 Lille, France (e-mail: bilaldaass@ gmail.com).
Spiegelhalter [6] and Granjon [7]. The EWMA control chart was introduced and developed by Roberts [8]. It was subsequently studied in many works. Examples are Hunter [9]; and Ye, Vilbert and Chen [10]. Numerous comparative studies are also presented in [11], [12]. In this respect, various tools, techniques, approaches and their applications in different fields have been developed [13], [14].

In this context, it is important to determine an appropriate value for the threshold used in these control charts. This value will determine the performance of the detection system. Indeed, by considering a too low threshold, we risk getting a large number of false alarms. On the other hand, a too high threshold leads to a detection delay in the best case, and a set of missed detections in the worst case. For example, if we consider the EWMA chart, the width of the control limits is usually defined as three times the standard deviation of the signal. The value of this threshold is somewhat arbitrary. In this sense, several studies have proposed an adaptation for change detection methods [15-17].

In our previous studies [18-21], an entropy-based strategy was proposed in order to overcome the constraint of using a fixed predetermined threshold. Moreover, we have discussed in [19] that the entropy-based adaptive threshold takes into account all previous observations. Thus, when several errors have occurred, the threshold can be significantly influenced. This problem has been addressed in [19] and a sliding time windowing technique was proposed. This technique consists in considering, at each iteration, only a window of the most recent observations. The window of length ' $n$ ' has been determined in [19] according to a method based on the minimization of the sum of the squared errors between the observations and the corresponding smoothed values. However, this method cannot work in a real-time manner as a preliminary learning step is necessary.

To address the need of fast and real-time sensor fault detection method, we extend our previous work by introducing a technique based on the use of Shannon's minimum conditional entropy. Thus, giving similar performance to the first one, but allowing moreover real-time operation without the necessity of the learning step. This paper is organized as follows. Section II briefly recalls the detection methodology based on the entropy-based criterion associated to two commonly used change detection techniques (Cusum and EWMA). Then, an improvement of this methodology using the

Denis Pomorski is with the University of Lille, CNRS, Centrale Lille, UMR 9189 CRIStAL - Centre de Recherche en Informatique, Signal et Automatique de Lille, F-59000 Lille, France (e-mail: denis.pomorski@univlille.fr).

Kamel Haddadi is with the University of Lille, CNRS, Centrale Lille, ISEN, University of Valenciennes, UMR 8520 - IEMN, F-59000 Lille, France (e-mail: kamel.haddadi@univ-lille.fr). 
sliding time window technique is presented in section III: a new method for real-time window length determination is implemented. Finally, section IV presents an experimental validation on real data from a mobile robotics platform.

\section{ADAPTIVE THRESHOLDING BASED ON THE ENTROPY CRITERION}

\section{A. Entropy-based criterion}

From a given phenomenon, the detection theory addresses the problem of discriminating a finite number of hypotheses. The knowledge of the vector of measurement $y=$ $\left(y_{1}, y_{2}, \ldots, y_{N}\right)$ issued from a vector of sensors $Y=$ $\left(Y_{1}, Y_{2}, \ldots, Y_{N}\right)$ allows to take a final decision $u$. In the context of the binary detection, this decision has two possible values $u \in\{0,1\}$, depending on whether true hypothesis $H$ is considered to be $H_{0}$ or $H_{1}$ "Fig. 1 ".

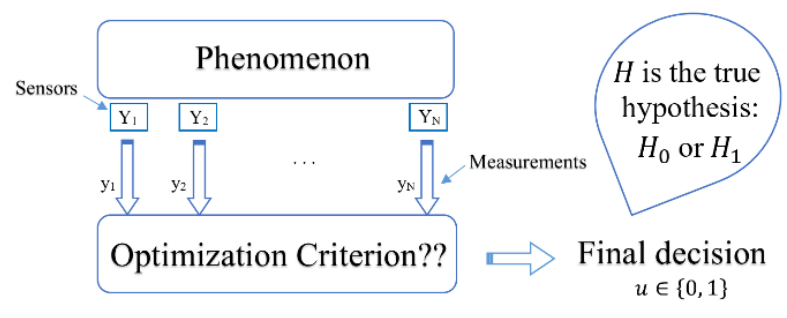

Figure 1. Principle of a binary detection system.

The optimization criterion chosen in this work is the minimization of the part of $H$ which is not explained by the final decision $u$. This quantity is defined by Shannon's conditional entropy $h(H / u)$ [20], [21], and represents the uncertainty on $H$ knowing the decision $u$. It can be written as a function of the probability of detection ' $P_{D}$ ' and the probability of false alarms ' $P_{F}$ ' as follows:

$$
h(H / u)=-\sum_{i \in\{0,1\}}\left[\alpha_{i} \log \frac{\alpha_{i}}{\alpha_{i}+\beta_{i}}+\beta_{i} \log \frac{\beta_{i}}{\alpha_{i}+\beta_{i}}\right]
$$

with:

$$
\left\{\begin{array}{cc}
\alpha_{1}=P_{0} P_{F} & \beta_{1}=\left(1-P_{0}\right) P_{D} \\
\alpha_{0}=P_{0}\left(1-P_{F}\right) & \beta_{0}=\left(1-P_{0}\right)\left(1-P_{D}\right)
\end{array}\right.
$$

$P_{0}$ is the prior probability of the hypothesis $H_{0}$. Minimizing $h(H / u)$ consists thus of minimizing a mean risk for which the costs are not constant, unlike the Bayesian criterion. Moreover, taking into account the following notation:

$$
\begin{cases}C_{00}=\log \frac{\alpha_{0}+\beta_{0}}{\alpha_{0}} & C_{10}=\log \frac{\alpha_{1}+\beta_{1}}{\alpha_{1}} \\ C_{01}=\log \frac{\alpha_{0}+\beta_{0}}{\beta_{0}} & C_{11}=\log \frac{\alpha_{1}+\beta_{1}}{\beta_{1}}\end{cases}
$$

And under the reasonable assumption that $C_{10}>C_{00}$ and $C_{10}>C_{11}$, the decision rule can be expressed as a likelihood ratio:

$$
\frac{P\left(u / H_{1}\right)}{P\left(u / H_{0}\right)} \underset{u=0}{\gtrless} \frac{P_{0}}{1-P_{0}} \times \frac{C_{10}-C_{00}}{C_{01}-C_{11}}=T h^{*}
$$

This structure is similar to that obtained using Bayes or Neyman-Pearson criterion. In this entropy-based approach, the costs are not constant but depend on the posterior probabilities.
In order to find the optimal value $T h^{*}$ of the threshold, an exhaustive search could be proposed. It consists of testing all possible values and keeping only the value minimizing $h(H / u)$. In most cases, and especially in a real-time context, a gradient-based technique is preferable [21].

\section{B. Control charts}

In the context of change detection, the Shewhart chart is very efficient when the magnitude of the shift is $1.5 \sigma$ to $2 \sigma$ or larger. Conversely, in the case of small shifts, the Cusum and EWMA control charts are good alternatives. However, these methods use predefined fixed thresholds, without any real justifications. We apply the variable threshold technique, based on the entropy, to these two algorithms.

\section{Cumulative sum - Cusum}

The Cusum chart directly integrates all the information into the sequence of sample values by plotting the cumulative sum of the deviations of the sample values from a target value.

Let $x_{k}$ be the $k^{t h}$ measurement. When the process is under control, $x_{k}$ is issued from a normal distribution with mean $\mu_{0}$ and standard deviation $\sigma$. The Cusum algorithm works with two statistics $C_{k}^{+}$and $C_{k}^{-}$. These statistics are called the Upper and Lower Cusum respectively:

$$
\left\{\begin{array}{c}
C_{k}^{+}=\max \left[0, x_{k}-\left(\mu_{0}+K\right)+C_{k-1}^{+}\right] \\
C_{k}^{-}=\max \left[0,-x_{k}+\left(\mu_{0}-K\right)+C_{k-1}^{-}\right]
\end{array}\right.
$$

with: $C_{0}^{+}=C_{0}^{-}=\mu_{0}$.

$K$ is usually called the reference value. It is often chosen halfway between the target $\mu_{0}$ and the mean $\mu_{1}$ :

$$
K=\frac{\left|\mu_{1}-\mu_{0}\right|}{2}
$$

The mean $\mu_{1}$ is the out-of-control value for which we consider that the process is out of control.

If either $C_{k}^{+}$or $C_{k}^{-}$exceeds the threshold $T h$, the process is considered out of control. A typical value of $T h$ is usually defined as five times the standard deviation $\sigma$ of the process.

\section{Exponentially Weighted Moving Average - EWMA}

The EWMA decision depends on the EWMA statistic, which is an exponentially weighted average of all previous data, including the most recent measurements. This decision is based on the weighting of the observations, so that the most recent observations contribute significantly.

The EWMA is sensitive to a small or gradual drift of the process. It is defined as follows:

$$
z_{k}=\lambda x_{k}+(1-\lambda) z_{k-1}
$$

With $z_{0}=\mu_{0}$ is the target, and $\lambda$ is the weighting factor.

The upper and lower control limits of the EWMA algorithm are defined as follows: 


$$
\left\{\begin{array}{l}
U C L_{k}=\mu_{0}+L \sigma \sqrt{\frac{\lambda}{(2-\lambda)}\left[1-(1-\lambda)^{2 k}\right]} \\
L C L_{k}=\mu_{0}-L \sigma \sqrt{\frac{\lambda}{(2-\lambda)}\left[1-(1-\lambda)^{2 k}\right]}
\end{array}\right.
$$

where $L$ is the width of the control limits. A typical value of $L$ is usually defined as three times the standard deviation $\sigma$ of the process.

\section{THE ADAPTIVE THRESHOLDING - SLIDING WINDOW TECHNIQUE}

The value of the threshold $T h$ used in the Cusum and EWMA charts is often determined without any real justification apart from the experiment. However, the value $T h$ retained chosen will condition the resulting performance.

The threshold can be learned automatically, at each step of the algorithm, using the entropy-based criterion [18]. We have demonstrated that this approach is much more robust (with respect to false alarms and missed detections) than the usual approach considering a fixed threshold. However, at each iteration, our adaptive threshold takes into consideration all previous observations. Consequently, when several model changes have occurred, the adaptive threshold can be significantly influenced. In order to solve this problem, we propose an improvement of our approach by using a sliding time windowing technique. The latter consists in defining a fixed window of length $n$. This implies that at each iteration, only the $n$ most recent observations are considered. In the following, we present two different methods that allow to determine the sliding time window length $n$.

\section{A. Smoothed value-based method}

The first method was proposed in [16]. It consists in defining the weighting factor of EWMA $\left(\lambda^{*}\right)$ as a first step. This is done by minimizing the sum of the squared errors (SSE) between the observations $x_{k}$ and the corresponding smoothed value $S_{k}$ calculated as follows:

$$
\operatorname{SSE}(\lambda)=\sum_{k=1}^{N_{O B S}}\left[S_{k}(\lambda)-x_{k}\right]^{2}
$$

where:

$$
S_{k}=\lambda x_{k-1}+(1-\lambda) S_{k-1}
$$

with $\mathrm{S}_{1}=x_{1}, \lambda \in\left[\begin{array}{ll}0 & 1\end{array}\right]$ and $N_{O B S}$ is the total number of observations. The optimal value of $\lambda$ is the one for which the sum of the squared error is minimal. The window length $n$ is therefore determined according to this formula:

$$
n=\left(2 / \lambda^{*}\right)-1
$$

In other words, this window is a standard moving average that approximates the exponential smoothing $\mathrm{S}$.

\section{B. Minimum conditional entropy based method}

According to the previous method, a learning step is required to determine $n$. To avoid this constraint, we propose a real-time method based on Shannon's conditional entropy minimization. This quantity, calculated by Eq. (1), increases very quickly, then stabilizes. The stability of the minimal Shannon's conditional entropy $h^{*}(H / u)$ seems to be a good criterion to determine the time-window needed to store a sufficient amount of information to minimize the uncertainty on $H$ knowing $u$.

The method is as follows: the time derivative of $h^{*}(H / u)$ is calculated according to (12) at each time step, and a violation occurs when this derivative at time step $k$ is less than a predefined precision value (13).

$$
\begin{gathered}
D h_{k}=\frac{h^{*}(H / u)_{k}-h^{*}(H / u)_{k-1}}{2} \\
\left|D h_{k}\right|<\left(1-\frac{\text { precision }}{100}\right) \times D h_{1} \rightarrow \text { violation }
\end{gathered}
$$

We set the value of the time-window length after a certain number of consecutive violations $K_{v}$.

$$
\sum_{p=1}^{k} 1_{\left\{\left|D h_{p}\right|<\left(1-\frac{\text { precision }}{100}\right) \times D h_{1}\right\}}>K_{v} \rightarrow n
$$

Although we cannot determine the optimal values of $K_{v}$ and precision, with some approximations, this method can give an acceptable result. This is done without waiting for the end of the procedure as in the smoothed value method. We can thus obtain an approximated value of the time-window length in real-time situations.

\section{APPLICATION IN COLLABORATIVE MOBILE ROBOTICS}

To validate our approach proposed, we consider an application consisting of two collaborative mobile robots (Turtlebot $^{\mathrm{TM}}$ ) [19]. The objective of the application is to accurately estimate the pose (i.e. position and orientation) of each robot. However, the information from the on-board sensors can be disturbed at any time (strong magnetic field, data loss, drift, etc.). Consquently, we need to detect and isolate faulty sensors in the most efficient way to exclude them from the pose estimation algorithm.

After describing the platform used, we define the residual on which the Cusum and EWMA algorithms are applied in order to detect sensor faults. And finally, the results obtained using the classical approach (with fixed thresholds) and those obtained by the approach proposed in section III (with adaptive thresholds without/with a sliding time-window) are compared.

\section{A. Platform description}

Each robot in the platform is equipped with two differential wheels and two freewheels to improve stability (Fig. 2). The angular and linear velocities of each robot are independently controlled by an on-board laptop computer using the Ubuntu/ROS system. This laptop communicates via Wi-Fi with a workstation equipped with Matlab ${ }^{\mathrm{TM}}$ software.

Each robot has wheel encoders, a gyroscope and an indoor navigation system (Marvelmind ${ }^{\mathrm{TM}}$ ). In addition, robot 1 is also equipped with a $2 \mathrm{D}$-laser scanner $\left(\mathrm{RPlidar}^{\mathrm{TM}}\right)$ and a camera (Kinect ${ }^{\mathrm{TM}}$ ). 


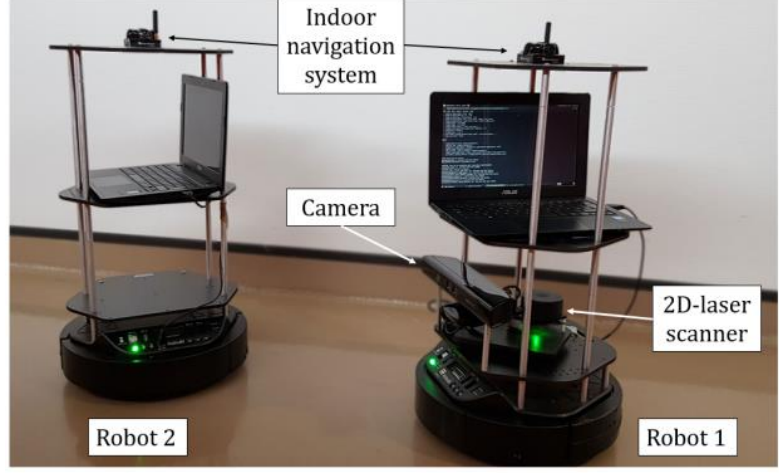

Figure 2. Mobile robotic platform.

\section{B. Residual definition}

At each step $k$, the state vector is defined as the pose of the two robots in a fixed global coordinate system:

$$
X_{k}=\left(\begin{array}{ll}
X^{1} & X^{2}
\end{array}\right)_{k}^{T}
$$

Where $X^{1}=\left(\begin{array}{lll}x^{1} & y^{1} & \theta^{1}\end{array}\right)$ and $X^{2}=\left(\begin{array}{lll}x^{2} & y^{2} & \theta^{2}\end{array}\right)$ are the pose of the robot 1 and 2, respectively. An EKF, detailed in [22], is applied to properly estimate the state vector. This filter operates in two steps: A prediction step based on an evolution model (e.g., an odometry model), and an update step based on sensor measurements.

When the robot moves, its wheel encoders are used to predict its pose using an odometry model. Moreover, at each instant, each robot gets observations from its own onboard sensors. In addition, robot 2 receives information from the Kinect camera embedded on robot 1 . In case of nominal operation (i.e. without any fault), these information are coherent. Nevertheless, in the case of a sensor failure, a drift of the estimation of the robot state compared to the real state will be observed. After detecting and excluding the faulty sensors, only the correct observations will be taken into account in the update step of the EKF.

A commonly used mismatch measure is the difference between the sensor observation $\left(Z_{k}\right)$ and the state prediction provided by the used evolution model $h\left(X_{k}\right)$. This measure, called the "residual", and on which an appropriate detection methodology is applied, will allow to exclude any erroneous observation from the fusion and estimation procedures. At each time step $k$, and for each observation, a residual is defined as follows:

$$
\operatorname{Res}_{k}=\left|Z_{k}-h\left(X_{k}\right)\right|
$$

The proposed change detection method applied to each component of $\operatorname{Res}_{k}$ allows for the detection of a significant difference between the raw measurement given by the sensor and the data issued from the predicted state. This faulty sensor is then excluded from the EKF. For each robot, at least one fault-free observation is assumed available at any time.

\section{Experimental results}

The experiment lasts 80 seconds, with a sampling time of 50 milliseconds. The robots follow a specific and defined trajectory while maintaining a distance of one meter between them.

In order to highlight the proposed approach, we introduced three different types of default that occur successively during the Kinect ${ }^{\mathrm{TM}}$ observation (a bias of $10 \mathrm{~cm}$ between iteration 500 and 700; a Gaussian noise " $\mathcal{N}(0 ; \sigma=0.10)$ " between iteration 900 and 1100; and a drift between iteration 1250 and 1500). In the following figure, we show the corresponding residual.

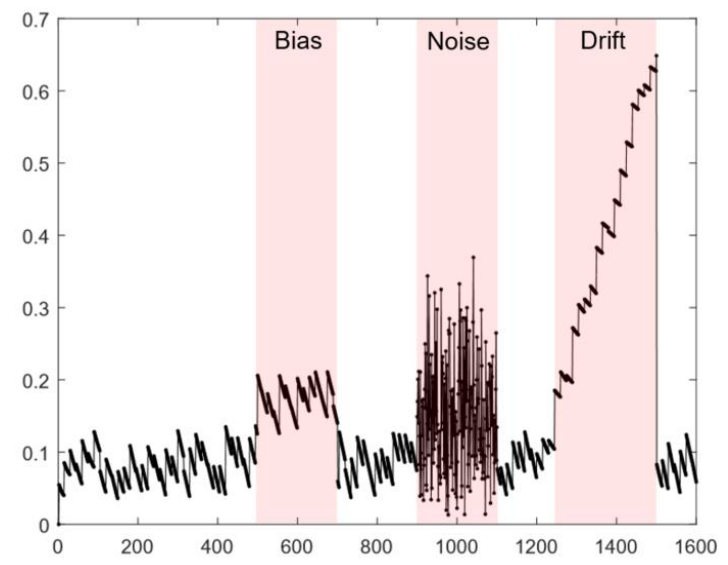

Figure 3. Corresponding residual as function of iteration number.

At each time step $k$, Cusum and EWMA techniques with/without adaptive threshold are applied to each component of the residual in order to detect the sensor faults. Fig. 4 shows the results obtained with the Cusum algorithm, using a fixed threshold (red) and using an adaptive threshold issued from the entropy-based criterion (green).

Similarly, Fig. 5 shows the obtained results with the EWMA method, using a fixed threshold (red) and using the adaptive threshold issued from entropy-based criterion (green).

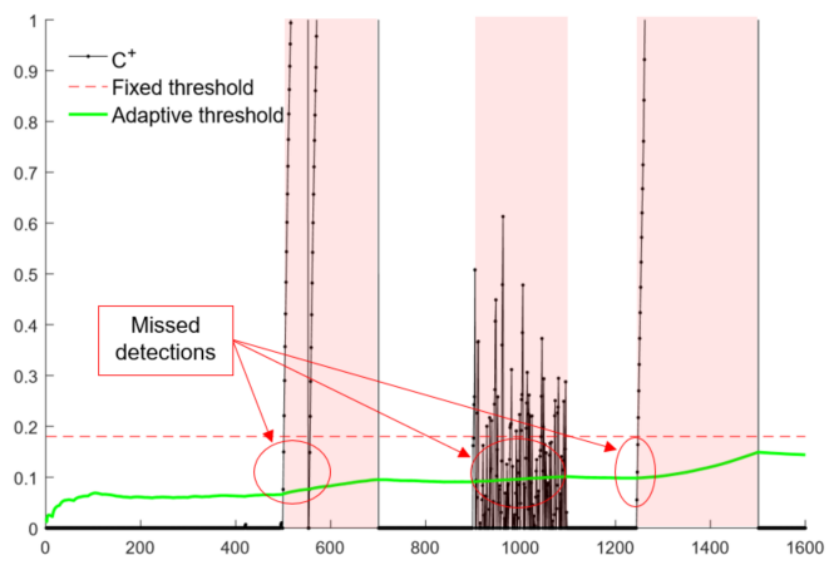

Figure 4. Cusum result using a fixed/adaptive threshold. 


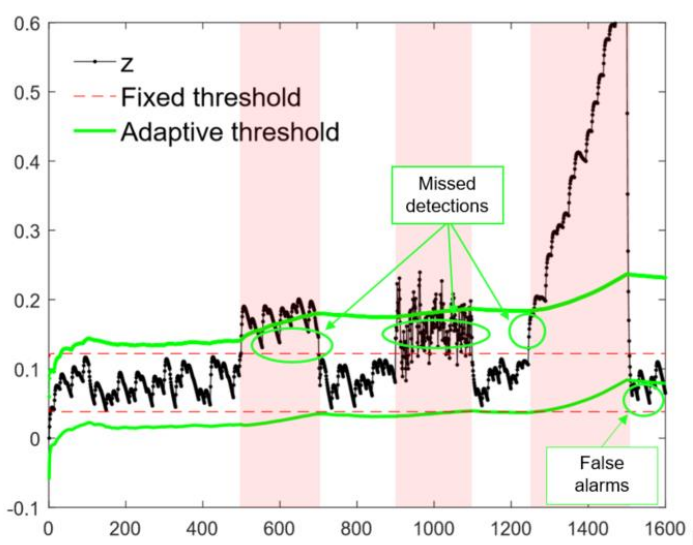

Figure 5. EWMA result using a fixed/adaptive threshold.

Note that the fixed threshold technique generates some missed detections (Fig. 4). We also note that after each default, the adaptive threshold is highly influenced, generating therefore false alarms and missed detections in Fig. 5. Indeed, this adaptive method needs time after each default in order to re-adjust the threshold. We apply therefore the sliding time-windowing technique of length $n$.

Since our application requires online, real-time operation without prior learning, the determination of the window length is performed using the minimum conditional entropy method. Fig. 6 presents the measure of Shannon's minimum conditional entropy applied to the residual andFig. 7" presents its temporal derivative $D h_{k}$.

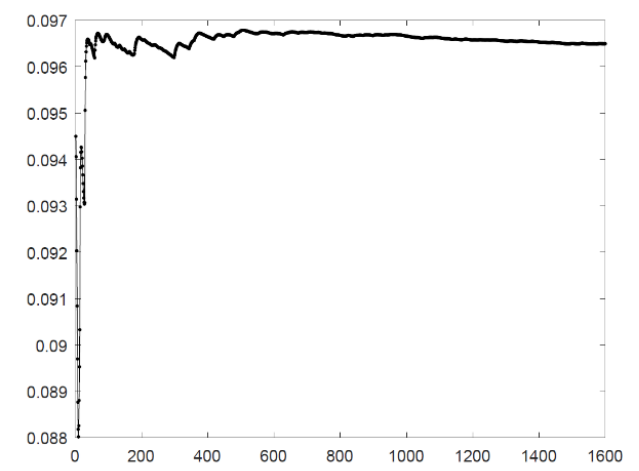

Figure 6. Shannon's minimum conditional entropy as a function of time.

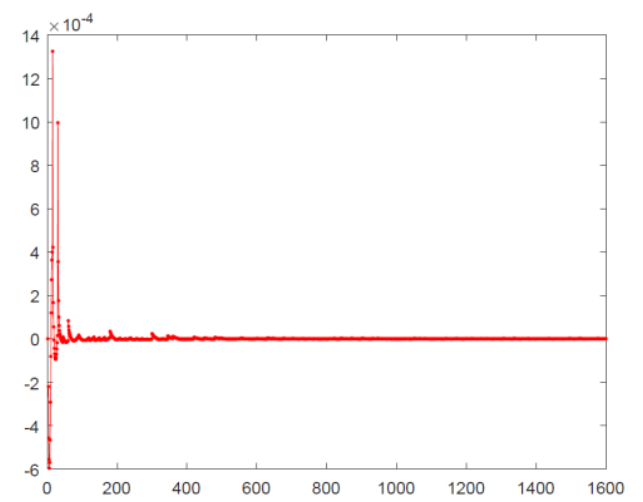

Figure 7. Time derivative of $h^{*}(H / u)$ as a function of time.
This stability of $h^{*}(H / u)$ is a good indication of how long it takes for the system to stop needing additional observations to refine the adaptive threshold. Fig. 8 presents the optimal (in the informational sense) length of the sliding window obtained as a function of precision (ranging from 0 to 99) and $K_{v}$ (ranging from 1 to 15 ).

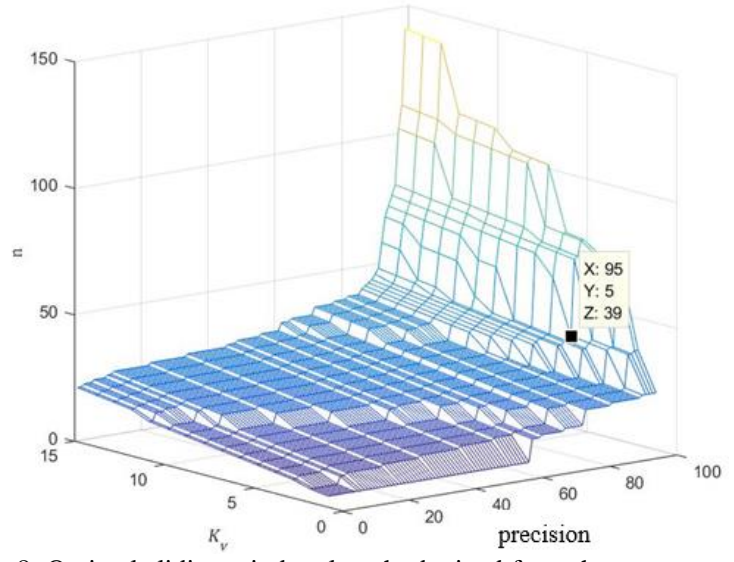

Figure 8. Optimal sliding window length obtained from the entropy method as a function of precision and $K_{v}$.

The optimal window length is an increasing function of the precision and $K_{v}$. Moreover, when the precision tends to 100 , it becomes difficult to have the required number of $K_{v}$ violations, the window length increases significantly. Therefore, in this application we have chosen the following values: $K_{v}=5$, precision $=95$. The optimal length of the temporal window obtained is therefore equal to 39.

We can notice that the window length determined online with the entropy method is very close to that determined after experimentation by the offline method based on the exponential smoothing ( $n=30$ in this case). However, we note that the offline method relies on the minimization of the sum of squared errors, a criterion that is difficult to justify as being the most appropriate.

We therefore propose to apply the sliding window whose length is obtained by the entropy based method. This technique is performed online, without prior learning. Fig. 9 (resp. Fig. 10) shows the results obtained with the Cusum (resp. EWMA) method with a fixed threshold on the one hand, and with an adaptive threshold derived from the entropy criterion and using the time window technique on the other hand.

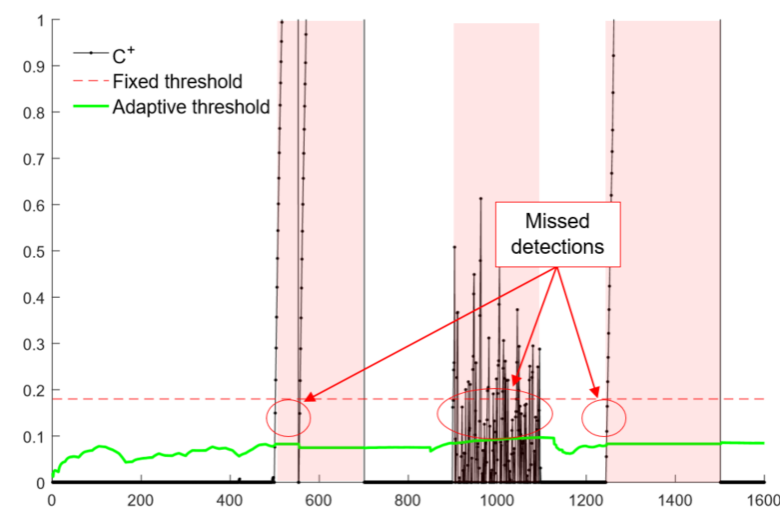

Figure 9. Cusum results using fixed / adaptive threshold with windowing - as a function of time. 


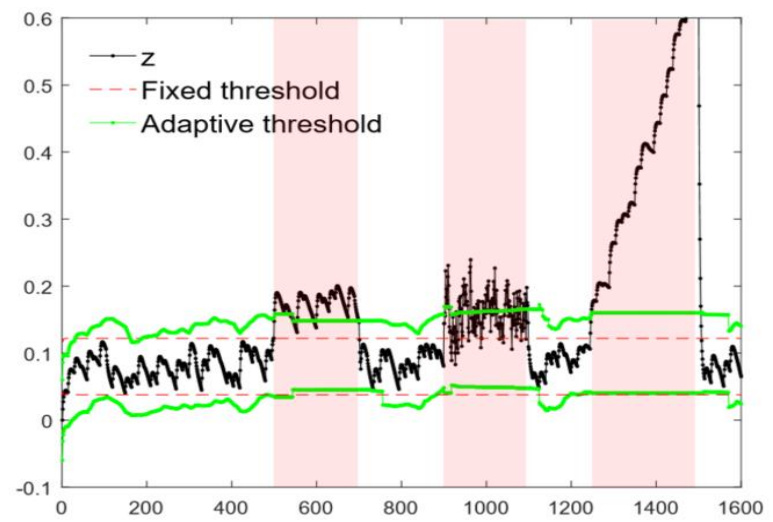

Figure 10. EWMA results using fixed / adaptive threshold with windowing as a function of time.

We note that the adaptive threshold without timewindowing is highly influenced after each default, which may implies some false alarms and missed detections (Fig. 4 and Fig. 5). However, by using the sliding time-windowing technique, the adaptive threshold $T h$ makes it possible to avoid these false alarms and missed detections (Fig. 9 and Fig. 10). Furthermore, the new threshold is more adaptive with respect to the residuals and it is not influenced by the defaults occurred previously.

\section{CONCLUSION}

In this paper, we have proposed to use a criterion based on Shannon's entropy to detect a model change in a digital sequence, in a general way. This digital sequence can be any kind of information delivered by a sensor. Our approach consists to determine an adaptive threshold that can be applied by any change detection method based on the (generalized) likelihood ratio. To illustrate our approach, we have combined this adaptive thresholding technique with two commonly used change detection methods: the Cusum and EWMA control charts.

However, the proposed approach consists, at each iteration, to take into account all previous observations, which can lead to a loss of robustness when several model changes have been occurred. To solve this problem, an improvement is proposed by using a sliding time-windowing technique: at each iteration of the algorithm, we consider only the $n$ most recent observations. Two methods have been identified to determine the value of the time-window length. The first method is based on the minimization of the sum of the squared errors between the measurements and the corresponding smoothed value. However, this method cannot work in real-time situations, a learning step is required. Therefore, we have proposed in this paper a second method based on Shannon's conditional entropy suited for real-time operation.

Finally, our approach makes it possible to propose a faulttolerant fusion methodology, which is illustrated by the use of an EKF in the context of collaborative mobile robotics. Both the standard change detection methods and the proposed approach are applied and compared to detect and remove faulty sensors. Our strategy is much more robust with respect to false alarms and missed detections. The proposed strategy has a significant robustness with respect to rare events, allowing especially its use, completely in real-time, in any change detection algorithm.

\section{REFERENCES}

[1] T. S. Lau and W. P. Tay, "Quickest Change Detection in the Presence of a Nuisance Change," IEEE Transactions on Signal Processing, vol. 67, no. 20, pp. 5281-5296, 2019.

[2] M. Awais and A. Haq, "New Shewhart-EWMA and Shewhart-CUSUM Control Charts for Monitoring Process Mean," Scientia Iranica, vol. 26, no. 6, pp. 3796-3818, 2019

[3] W.A. Shewhart, Statistical Method from the Viewpoint of Quality Control. Washington: Graduate School, Department of Agriculture, 1939.

[4] E.S. Page, "Continuous Inspection Schemes," Biometrika, vol 41, no. $1 / 2$, pp. 100-115, 1954.

[5] M. Basseville and I.V. Nikiforov, Detection of Abrupt Changes: Theory and Application. Englewood Cliffs, NJ: Prentice-Hall (104), 1993.

[6] O. A. Grigg, V. T. Farewell and D. J. Spiegelhalter, "Use of Riskadjusted CUSUM and RSPRTcharts for Monitoring in Medical Contexts," Stat Methods Med Res, vol. 12, no. 2, pp. 147-170, Apr. 2003.

[7] P. Granjon, The CuSum Algorithm-A Small Review. Technical Report, 2013.

[8] S. W. Roberts, "Control Chart Tests Based on Geometric Moving Averages," Technometrics, vol. 1, no. 3, pp. 239-250, Aug. 1959.

[9] J. S. Hunter, "The Exponentially Weighted Moving Average," Journal of Quality Technology, vol. 18, no. 4, pp. 203-210, 1986.

[10] N. Ye, S. Vilbert and Q. Chen, "Computer Intrusion Detection Through EWMA for Autocorrelated and Uncorrelated Data," IEEE Transactions on Reliability, vol. 52, no. 1, pp. 75-82, 2003.

[11] N. Boudaoud and Z. Cherfi, "A Comparative Study of CUSUM and EWMA Charts: Detection of Incipient Drifts in a Mutlivariate Framework," Quality Engineering, vol. 17, no. 4, pp. 703-709, 2005.

[12] D. M. Hawkins and Q. Wu, "The CUSUM and the EWMA Head-tohead," Quality Engineering, vol. 26, no. 2, pp. 215-222, 2014.

[13] S. Ottenstreuer, C. H. Weiß and S. Knoth, "A Combined ShewhartCUSUM Chart with Switching Limit," Quality Engineering, vol. 31, no. 2, pp. 255-268, 2019.

[14] A. Haq, J. Brown and E. Moltchanova, "Improved Fast Initial Response Features for Exponentially Weighted Moving Average and Cumulative Sum Control Charts," Quality and Reliability Engineering International, vol. 30, no. 5, pp. 697-710, 2014.

[15] G. Verdier, N. Hilgert et J.-P. Vila, "Calcul d'un seuil adaptatif pour des algorithmes de type CUSUM,” 37èmes Journées de Statistique, Pau, 2005.

[16] P. Cisar and S. M. Cisar, "EWMA Statistic in Adaptive Threshold Algorithm," in $200711^{\text {th }}$ International Conference on Intelligent Engineering Systems, 2007, pp. 51-54.

[17] D. Lee and K. Akatsu, "An Improved Position Sensor Fault Detection and Algorithm Transition using Adaptive Threshold for Sensorless Control of IPMSM," in IECON 2019 - 45 th Annual Conference of the IEEE Industrial Electronics Society, vol. 1, Oct. 2019, pp. 910-915.

[18] B. Daass, D. Pomorski and K. Haddadi, "Using an Adaptive Entropybased Threshold for Change Detection Methods - Application to FaultTolerant Fusion in Collaborative Mobile Robotics," in $20196^{\text {th }}$ International Conference on Control, Decision and Information Technologies (CoDIT), Paris, France, 2019, pp. 1173-1178.

[19] B. Daass, D. Pomorski and K. Haddadi, "An Adaptive Threshold for Change Detection Methods using a Windowed Entropy-based Criterion - Application to Fault-tolerant Fusion in Collaborative Mobile Robotics," in $20194^{\text {th }}$ Conference on Control and Fault Tolerant Systems (SysTol), Casablanca, Morocco, 2019, pp. 56-61.

[20] I. Y. Hoballah and P. K. Varshney, "An Information Theoretic Approach to the Distributed Detection Problem," IEEE Transactions on Information Theory, vol. 35, no. 5, pp. 988-994, Sept. 1989.

[21] D. Pomorski, "Entropy-based Optimisation for Binary Detection Networks," in Proceedings of the Third International Conference on Information Fusion, vol. 2, Jul. 2000, p. THC4/3-THC410 vol.2.

[22] M. S. Grewal and A. P. Andrews, "Applications of Kalman Filtering in Aerospace 1960 to the Present [Historical Perspectives]," IEEE Control Systems Magazine, vol. 30, no. 3, pp. 69-78, Jun. 2010. 